$\$$ Research Square

\title{
The Risk of Dementia in Adults with Abdominal Aortic Aneurysm
}

\author{
Hyung-jin Cho \\ Eunpyeong St. Mary' Hospital \\ Ju-hwan Yoo \\ The Catholic University of Korea \\ Mi-hyeong Kim \\ Eunpyeong St. Mary' Hospital \\ Kyung-jai Ko \\ Kangdong Sacred Heart Hospital \\ Kang-woong Jun \\ Bucheon St. Mary's Hospital \\ Kyung-do Han \\ Soongsil University \\ Jeong-kye Hwang ( $\nabla_{\text {jjungyong@catholic.ac.kr ) }}$ \\ Eunpyeong St. Mary' Hospital
}

\section{Research Article}

Keywords: Aortic aneurysm, Abdominal, Dementia, Vascular, Alzheimer Disease.

Posted Date: September 8th, 2021

DOI: https://doi.org/10.21203/rs.3.rs-870520/v1

License: (c) (1) This work is licensed under a Creative Commons Attribution 4.0 International License. Read Full License

Version of Record: A version of this preprint was published at Scientific Reports on January 24th, 2022. See the published version at https://doi.org/10.1038/s41598-022-05191-1. 


\section{Abstract}

\section{Background}

Abdominal aortic aneurysm (AAA) and dementia have similar epidemiological profiles and common pathogenic mechanisms. However, there have been few studies on the link between these two diseases.

\section{Materials and methods}

For this study, information from 2009 to 2015 was extracted from the Korean National Health Insurance system database. A total of 15,251 participants with a new diagnosis of AAA was included. Propensity score matching by age and sex with patients in whom AAA was not diagnosed was used to select the control group of 45,753 participants. The primary endpoint of this study was newly diagnosed dementia (Alzheimer's disease (AD), vascular dementia (VD), or other type of dementia).

\section{Results}

The incidence of dementia was 23.084 per 1000 person years in the AAA group, which was higher than that of the control group (15.438 per 1000 person years). When divided into AD and VD groups, the incidence of AD was higher than that of VD, but the HR of AAA for occurrence of dementia was higher in VD (1.382 vs. 1.784). Among the various risk factors, there was an interaction if age, hypertension, and history of cardiovascular disease with incidence of dementia $(p<0.05)$. In the presence of hypertension, the HR for occurrence of dementia was high according to presence or absence of AAA (1.474 vs 1.165). In addition, this study showed higher HR in the younger age group (age $<65)$ and in the group with no history of cardiovascular disease [1.659 vs. 1.403 (age), 1.521 vs. 1.255 (history of cardiovascular disease)].

\section{Conclusions}

AAA was associated with increased risk of dementia regardless of $A D$ or VD, even after adjusting for several comorbidities. These findings indicate that follow-up with AAA patients is necessary for early detection of signs and symptoms of dementia.

\section{Article Highlights}

\section{Type of Research}

We performed population-based cross-sectional study using Korean National Health Insurance system database. A total of 15,251 participants with a new diagnosis of Abdominal aortic aneurysm (AAA) was included. Propensity score matching by age and sex with patients in whom AAA was not diagnosed was used to select the control group of 45,753 participants.

\section{Key Findings}

The incidence of dementia was 23.084 per 1000 person years in the AAA group, which was higher than that of the control group (15.438 per 1000 person years). When adjusting comorbidities, the incidence rate remained significantly higher in the AAA group; and the hazard ratio was 1.422 .

\section{Take Home Message}

This study suggests that follow-up with AAA patients is necessary for early detection of signs and symptoms of dementia, regardless of Alzheimer dementia or vascular dementia. In particular, surveillance is necessary when high blood pressure accompanies AAA or if AAA occurs in the absence of history of heart disease in a patient younger than 65 years.

\section{Table of Contents Summary}

In the population-based cross-sectional study, it was found that the incidence rate of dementia was significantly higher in the AAA group, even when adjusting several comorbidities. Therefore, early detection of signs and symptoms of dementia in the AAA patients is important.

\section{Introduction}

Dementia is acquired loss of cognition in multiple cognitive domains that is sufficiently severe to affect social or occupational function.[1] The pooled point prevalence of dementia per 1000 in 23 community setting studies was 48.62, and the incidence rate of dementia per 1000 personyears was 17.18 in the community-only setting. The three most common types of dementia in order of prevalence are Alzheimer's disease (AD), vascular dementia (VD), and Lewy body dementia.[2] Age is the most important risk factor for dementia, with prevalence doubling every 5 years after age 65 ; the prevalence is increased from $2-3 \%$ in those $65-69$ years to $30 \%$ among individuals older than 80 .[3]

An abdominal aortic aneurysm (AAA) is a permanent localized dilatation of the abdominal aorta that exceeds the normal diameter by $50 \%$, or $>3$ $\mathrm{cm}$. The mean annual incidence of new AAA diagnoses in Western populations is $0.4-0.67 \%$.[4] Age is a risk factor for AAA, and there are

Page 2/10 
similarities in epidemiological profiles between dementia and AAA including smoking, hypertension, obesity, and hyperlipidemia.[2, 4, 5]

Until recently, dementia has been considered neither preventable nor treatable, but recent clinical trials assessing the effects of lifestyle modification in dementia offer hope, showing that $35 \%$ of $A D$ risk is modifiable.[2,6] These modifiable factors generally do not apply to $A A A$; however, given the similarity of their epidemiological profiles and the many common pathogenic mechanisms,[7] the possibility of a link between the two can be considered.

Therefore, in this study, we investigated the effects of the presence of AAA on the incidence rate of dementia during a 7-year period in Korean using a Korean National Health Insurance Service (NHIS) database.

\section{Method}

\subsection{Data Source}

The Korean National Health Insurance (NHI) system consists of two major health care programs for universal coverage of all residents of Korea: $\mathrm{NHI}$ and Medical Aid (MA). Approximately $97 \%$ of the population is covered by NHI, and the remaining $3 \%$ is covered by MA.[8] The NHIS provides biannual health examinations for all insured Koreans and maintains an extensive data set of Koreans that includes patient demographics, medical treatment and procedures, and disease diagnoses according to the International Classification of Disease, 10th editions (ICD-10). For this study, information from 2009 to 2015 was extracted from the NHIS database

\subsection{Sampled Patients}

Patients in the database who were newly diagnosed with AAA between 2009 and 2015 were enrolled $(n=45,767)$. The AAA group was defined as patients diagnosed with AAA codes (171.3-171.6, I71.8, and 171.9) more than twice at an outpatient department in the past year, who had been hospitalized with one of these AAA codes more than once, or who had undergone aneurysm repair surgery such as open surgical aneurysm repair (OSAR) or endovascular aneurysm repair (EVAR) defined by with the codes ( 00223, 00224, 00234, M6611, and M6612). We excluded patients who had not undergone a health examination within 2 years prior to diagnosis of AAA $(n=26,121)$, were younger than 40 years $(n=431)$, had data missing $(n=250)$, or had a previous diagnosis of dementia $(n=1024)$. Among the remainder, a group of patients who were followed for more than one year was selected $(n=15251)$. To select the control group, with a number of participants three times larger than that of the patient group ( $n=$ 45753), we used propensity score matching by age and sex with patients in whom AAA was not diagnosed (Fig. 1). The primary endpoint of this study was newly diagnosed dementia (AD, VD, or other type of dementia).

\subsection{Data Collection and Definition}

We collected baseline data from the NHIS database of age, sex, smoking status, alcohol consumption, physical activity, waist circumference, body mass index (BMI), and income level. Data about comorbidities, including hypertension, diabetes, dyslipidemia, chronic kidney disease (CKD), and history of cardiocerebrovascular disease (CVD), also were collected. Smoking status was classified as non-smoker, ex-smoker, and current smoker. Alcohol consumption was classified as heavy drinker when the average daily alcohol intake was $30 \mathrm{~g}$ or more, mild to moderate drinker when the individual consumed less than $30 \mathrm{~g}$ daily, and non-drinker. Physical activity was divided into two groups, one performing moderate exercise more than 5 days per week or vigorous exercise more than 3 days per week; and all others were classified into the second group. Income level was classified into a group that received medical aid and was in the bottom $20 \%$ of income and a group of all others. Comorbidities were defined as follows: DM codes [(E11-14 with antidiabetic medication) or (fasting blood sugar $\geq 126)$ ], hypertension [codes (I10-13 and I-15 with antihypertensive medication) or (SBP $\geq 140$ ) or (DBP $\geq 90)$ ], dyslipidemia [(code E78 with antihyperlipidemic medications) or (total cholesterol $\geq$ 240)], CKD (eGFR <60), and history of CVD [cerebrovascular disease codes(I60-64) and cardiovascular disease codes (I20-25)]. Finally, dementia was defined as follows; AD [code (F00, and G30)], VD [code (F01)], other dementia [code (F02, F03, and G31)] with one or more medication for dementia (donepezil, rivastigmine, galantamine, or memantine). When there were codes for both AD and VD, the main diagnosis was selected as the final diagnosis. If there was neither AD nor VD as a main diagnosis up to the second claim database, the diagnosis was defined as "other dementia". This study is approved by the Institutional Review Board of The Catholic University of Korea, Eunpyeong St. Mary's Hospital, Seoul, Korea (approval no. PC20ZISI0145). All methods were performed in accordance with the relevant guidelines and regulations approved by the Institutional Review Board of The Catholic University of Korea, Eunpyeong St. Mary's Hospital, Seoul, Korea. Informed consent was waived because all analysis used anonymous data.

\subsection{Statistical Analysis}

Continuous variables are presented as mean \pm standard deviation, and categorical variables are presented as number (percentage). To compare characteristics between patient and control groups, Student's t-test was used for continuous variables and the chi-square test or Fisher's exact test for categorical variables. The incidence rates of dementia are presented per 1000 person-years. Multivariate Cox regression models were used to evaluate the association of absence or presence of AAA with incidence of new-onset dementia. Model 1 examined the unadjusted hazard ratios (HRs). Model 2 was adjusted for age; sex; income level; and presence of diabetes, hypertension, or dyslipid11emia. In Model 3, smoking status, alcohol consumption, exercise status, and BMI were added; and history of CVD was added to Model 4 . The cumulative incidence of dementia 
according to presence of AAA was calculated using Kaplan-Meier curves; the log-rank test was performed to analyze differences among the groups. Statistical significance was set at $p<0.05$. All statistical analyses were performed using SAS version 9.4 (SAS Institute Inc., Cary, NC, USA.) and the R Project for Statistical Computing version 3.3.

\section{Results}

\subsection{Baseline characteristics according to presence of AAA}

The characteristics of subjects by presence of AAA are presented in Table 1. Diabetes, hypertension, and dyslipidemia are known risk factors for AAA and were more common in the AAA group. Also, there were more smokers in the AAA group, and BMI and abdominal circumference were higher in the AAA group. In addition, history of CVD was significantly higher in the AAA group, but alcohol consumption and exercise level were lower in the AAA group.

Table 1

Clinical characteristics of control and abdominal aortic aneurysm (AAA) patients.

\begin{tabular}{|c|c|c|c|}
\hline \multirow[b]{2}{*}{ Variables } & \multicolumn{2}{|l|}{ AAA } & \multirow[t]{2}{*}{ p-value } \\
\hline & No & Yes & \\
\hline & 45753 & 15251 & \\
\hline Age & $66.08 \pm 10.14$ & $66.08 \pm 10.14$ & 1 \\
\hline Sex, male & $29874(65.29)$ & $9958(65.29)$ & 1 \\
\hline Smoking status & & & $<.0001$ \\
\hline Non & $26776(58.52)$ & $7641(50.1)$ & \\
\hline Ex & $10772(23.54)$ & $3666(24.04)$ & \\
\hline Current & 8205(17.93) & $3944(25.86)$ & \\
\hline Alcohol consumption & & & $<.0001$ \\
\hline None & $28530(62.36)$ & 10207(66.93) & \\
\hline Mild to moderate & 14181(30.99) & 4087(26.8) & \\
\hline Heavy & $3042(6.65)$ & $957(6.27)$ & \\
\hline Regular exercise & 10473(22.89) & $3147(20.63)$ & $<.0001$ \\
\hline Diabetes mellitus & $9059(19.8)$ & $3196(20.96)$ & 0.002 \\
\hline Hypertension & 24564(53.69) & 12072(79.16) & $<.0001$ \\
\hline Dyslipidemia & $15050(32.89)$ & $8827(57.88)$ & $<.0001$ \\
\hline Income level, Low & 8683(18.98) & 2799(18.35) & 0.0872 \\
\hline BMI & $23.93 \pm 3.04$ & $24.05 \pm 3.15$ & $<.0001$ \\
\hline Waist circumference & $83.19 \pm 8.48$ & $84.23 \pm 8.78$ & $<.0001$ \\
\hline SBP & $127.45 \pm 15.41$ & $128.52 \pm 16.49$ & $<.0001$ \\
\hline DBP & $77.37 \pm 9.89$ & $78.34 \pm 10.72$ & $<.0001$ \\
\hline Glucose & $104.26 \pm 27.5$ & $102.33 \pm 25.39$ & $<.0001$ \\
\hline Cholesterol & $193.03 \pm 40.19$ & $192.93 \pm 43.44$ & 0.7955 \\
\hline History of cerebrovascular disease & 2512(5.49) & 2348(15.4) & $<.0001$ \\
\hline $\begin{array}{l}\text { History of } \\
\text { cardiovascular disease }\end{array}$ & $5653(12.36)$ & $7762(50.9)$ & $<.0001$ \\
\hline
\end{tabular}

\subsection{Incidence of dementia}


The incidence of dementia was 23.084 per 1000 person years in the AAA group, which was higher than that of the control group. When all models were applied respectively, the incidence rate remained significantly higher in the AAA group; and the hazard ratio was $1.516,1.625,1.568$, and 1.422 for models 1 through 4, respectively. When divided into AD and VD groups, the incidence of AD was higher than that of VD, but the HR of AAA for dementia was higher in the VD group (1.382 vs. 1.784 in model 4). (Table 2).

Table 2

Hazard ratio of AAA for incidence of dementia

\begin{tabular}{|c|c|c|c|c|c|c|c|c|}
\hline AAA & $\mathbf{N}$ & Dementia & Duration & Rate & Model 1 & Model 2 & Model 3 & Model 4 \\
\hline & & All & & & & & & \\
\hline No & 45753 & 3234 & 209489.14 & 15.4376 & 1 & 1 & 1 & 1 \\
\hline \multirow[t]{2}{*}{ Yes } & 15251 & 1432 & 62034.29 & 23.084 & $1.516(1.424,1.613)$ & $1.625(1.523,1.734)$ & $1.568(1.468,1.674)$ & $1.422(1.326,1.525)$ \\
\hline & & $A D$ & & & & & & \\
\hline No & 45753 & 2611 & 209489.14 & 12.4637 & 1 & 1 & 1 & 1 \\
\hline \multirow[t]{2}{*}{ Yes } & 15251 & 1090 & 62034.29 & 17.5709 & $1.433(1.335,1.538)$ & $1.548(1.438,1.666)$ & $1.495(1.388,1.61)$ & $1.382(1.277,1.496)$ \\
\hline & & VD & & & & & & \\
\hline No & 45753 & 332 & 209489.14 & 1.58481 & 1 & 1 & 1 & 1 \\
\hline Yes & 15251 & 213 & 62034.29 & 3.43358 & $2.18(1.835,2.59)$ & $2.233(1.865,2.672)$ & $2.136(1.781,2.561)$ & $1.784(1.467,2.169)$ \\
\hline
\end{tabular}

AAA: Abdominal aortic aneurysm, AD: Alzheimer's disease, VD: Vascular dementia

Model 1: adjusted for age and sex, Model 2 : adjusted for age, sex, income level, diabetes, hypertension, and dyslipidemia. Model 3: adjusted for age, sex, income level, diabetes, hypertension, dyslipidemia, smoking status, alcohol consumption, exercise status, and BMI. Model 4: adjusted for age, sex, income level, diabetes, hypertension, dyslipidemia, smoking status, alcohol consumption, exercise status, BMI, and history of CVD.

The Kaplan-Meier plot shows a comparison with the control group. All types of dementia occurred more frequently in the AAA group, which also was observed when divided into AD and VD groups (log rank test p value <0.001) (Fig. 2).

\subsection{Interactions with AAA on occurrence of dementia}

Among the factors, there was interaction of age, hypertension, and history of cardiovascular disease with AAA $(p<0.05)$. In the presence of hypertension, the HR for occurrence of dementia was high according to presence or absence of AAA (1.474 vs 1.165). These results were observed in the younger age group and in the group with no history of cardiovascular disease [1.659 vs. 1.403 (age), 1.521 vs. 1.255 (history of cardiovascular disease)] (Supplemental Table 3).

\section{Discussion}

The current social expenditure of dementia is US\$ 604 billion/ year worldwide, and it is projected that the number of dementia patients will triple by 2050. Therefore, it is possible to estimate the global socio-economic impact of dementia.[6,9] For treatment of dementia, a variety of pharmacologic and non-pharmacologic approaches has been attempted, but their efficacy has not been confirmed. Rather, treatment is focused on symptom control, and prevention is considered the top priority.[1, 2, 6, 10] A recent review by Livingston and coworkers suggests that approximately $35 \%$ of dementias is attributable to nine modifiable risk factors.[2] To prevent dementia, it is necessary to manage these risk factors including diabetes, hypertension, smoking, and obesity.

Each risk factor affects dementia through a variety of mechanisms. A precise mechanism between diabetes and dementia has not been reported in human research but has been suggested through animal models. Such mechanisms include cerebral insulin resistance, accumulation of glycation end products, and inflammation.[11, 12] Several mechanisms are suggested between hypertension and dementia. Hypertension causes vessel wall remodeling and endothelial dysfunction, which result in an autoregulatory deficit. Due to these neurological changes, the brain is vulnerable to hypoperfusion resulting from decrease in systemic blood pressure.[13] This decrease in cerebral blood flow affects dementia through direct damage to the brain, such as a decrease in hippocampal volume. However, further research is needed to elucidate the mechanisms that directly act on cognitive decline.[14] In cases of obesity, dementia is affected by both distinct and overlapping physiological changes, such as increased central inflammation, oxidative stress, sleep apnea, decreased neurogenesis, and silent infarcts. These can directly impact brain function.[15] Smoking can contribute secondarily by causing diseases considered risk factors for AD such as DM, CVD, hypertension and dyslipidemia. Also, smoking generates oxidative stress, leading to pathological $\beta$-amyloid $(A \beta)$ processing, tau protein hyperphosphorylation, and mild cerebrovascular dysfunction.[16] 
Unlike in dementia, DM shows a protective effect in AAA, and the mechanisms are the activity of glycation and cross-linking on ECM remodeling and the anti-inflammatory properties of the TGF- $\beta$ signaling pathway, as well as the impact of intraluminal thrombus (ILT) formation, neoangiogenesis, and vascular smooth muscle cell (VSMC) apoptosis. Also, antidiabetic drugs such as metformin, sulfonylurea, and thiazolidinedione interfere with the pathophysiological mechanisms of AAA.[17] In hypertension, a meta-analysis demonstrated an association with dementia. However, DBP is more highly correlated with dementia than is SBP, and further studies are needed to identify the precise mechanism. $[4,18]$ The mechanism of the relationship between obesity and dementia can be explained by the following mechanism. Interleukin-18 (IL18) uses both the IL18 receptor (IL18r) and the Na-Cl co-transporter (NCC) to promote AAA formation. Lesion adipocyte and perivascular adipose tissues contribute to AAA pathogenesis by releasing leptin and FABP4 to induce IL18, IL18r, and NCC expression and promote IL18 activity.[19] To date, no causative link has been proven between smoking and AAA formation [4]; however, some studies suggest that the effect of tobacco smoke on AAA development is related to altered inflammatory cell function acting to enhance matrix damage through MMP-independent pathways.[20]

While AAA and dementia share risk factors, there are both commonalities and differences in the mechanisms by which the factors act on the disease. In addition, there is a lack of research on the mechanisms of risk factors related to AAA. As the association between dementia and AAA was verified through this study, the mechanisms by which risk factors act on AAA in dementia must be studied.

In this study, even when the effects of the common risk factors of the two diseases were excluded, there was a relationship between AAA and dementia. These associations can be estimated by examining the pathophysiology of AAA.

The first such association is related to atherosclerosis. Traditionally, it was thought that AAA develops as a pathological response to aortic atherosclerosis. Until half a century ago, the term "atherosclerotic aneurysm" was used. This view still is favored by some researchers.[21, 22] Atherosclerotic plaque growth leads to a compensatory arterial response. In other words, due to arterial narrowing, hemodynamic changes such as those in shear stress occur. The endothelium detects this and changes the phenotype of vascular smooth muscle cells, allowing remodeling through secretion of proteolytic enzymes such as metalloproteinase (MMP).[21-23] Atherosclerosis typically is widespread throughout the vasculature, and the carotid artery is not exempt.[24] Carotid atherosclerosis and stiffness consequently cause brain microcirculation transformation and increase blood-brain barrier (BBB) permeability, leading to cognitive impairment.[25] Similarly, associations between descending thoracic aortic plaque and acceleration of brain aging have been demonstrated, and trends in accelerated progression of brain atrophy and progression of cerebrovascular lesions have been reported.[26, 27]

The second association is inflammation and matrix degradation. Aortic inflammation is believed to lead to destruction of aortic media and vascular smooth muscle cell apoptosis and dysfunction. Release of a range of proteolytic enzymes, such as MMP and cysteine proteases, produces reactive oxygen, cytokines, and related products.[21] The tissue inhibitors of MMP (TIMP) are increased in the wall of the aneurysm[28]; however, the balance between TIMP and MMP seems to favor proteolysis,[29] and elastin and collagen fibers are degraded. Also, due to the paracrine effect of VSMC, a protective effect is needed to maintain homeostasis from proteolysis and inflammatory reactions.[30] However, VSMC apoptosis prevents this protective effect. These MMPs (MMP-2) induce breakdown of the BBB, disrupt oxidative homeostasis in AD,[31] and play a role in the impaired $A \beta$ peptide metabolism responsible for progression of dementia.[32] Reactive oxygen species (ROS) affect $A D$ by inducing oxidation of lipids, proteins, and nucleic acids and impairing A $\beta$ clearance by the low density lipoprotein receptor-related protein (LPR1) through its oxidation.[33]

The hazard ratio of AAA for dementia in this study was 1.422, and the reason for this was deduced based on the mechanisms of AAA occurrence. However, since this deduction is only a possibility, further research is needed. Also, as documented in Supplemental Ttable 3 , there were interactions of age, hypertension, and history of cardiovascular disease with AAA. The HR of dementia was high according to the presence or absence of AAA in the presence of hypertension, in the younger age group, and in the group with no history of cardiovascular disease. In this study, all of these factors were related to dementia, but there were differences in the directions of the interactions. Synergy was present with hypertension because it uniquely influenced dementia with AAA. However, other factors influenced the occurrence of dementia in various ways, indicating a common denominator with AAA. As a result, the HR of AAA for dementia was decreased statistically. This, too, will require further research.

The present study has several limitations. First, this study was a retrospective analysis. To overcome this, the influence of each variable was adjusted through a multivariate logistic regression model. However, control of the confounders among variables was not achieved. Second, diagnosis of AAA and dementia and identification of other risk factors solely were based on diagnostic codes, possibly introducing bias. Third, considering the time of onset, a rough causal relationship might be inaccurately inferred because of the it is a retrospective nature of the study. Also, the degree of cognitive impairment and issues such as AAA diameter are not known, so the correlation between these could not be demonstrated clearly.

However, our study also has a number of strengths. First, we believe that this is the first study to not only demonstrate the relationship between AAA and dementia, but also to illustrate the effect of AAA on dementia in relation to each risk factor. Second, this study used a large, national sample with a relatively long follow-up period. 


\section{Conclusions}

We found that AAA was associated with increase in the risk of dementia regardless of AD or VD, even after adjusting for several comorbidities. These finding indicate that continued follow-up is necessary in AAA patients to permit early detection of signs and symptoms of dementia. In particular, surveillance is necessary when high blood pressure accompanies AAA or if AAA occurs in the absence of history of heart disease in a patient younger than 65 years.

\section{Declarations}

\section{Author Disclosures}

All authors have nothing to disclose.

\section{Declaration of conflicting interest}

None declared

\section{Acknowledgements}

This research was supported by a grant funded by The Catholic University of Korea, Eunpyeong St. Mary's Hospital, Research Institute of Medical Science in program year 2020.

\section{References}

1. Arvanitakis Z, Shah RC, Bennett DA (2019) Diagnosis and Management of Dementia: Review. JAMA 322:1589. https://doi.org/10.1001/jama.2019.4782

2. Livingston G, Sommerlad A, Orgeta V, et al (2017) Dementia prevention, intervention, and care. The Lancet 390:2673-2734. https://doi.org/10.1016/S0140-6736(17)31363-6

3. Fiest KM, Jetté N, Roberts Jl, et al (2016) The Prevalence and Incidence of Dementia: a Systematic Review and Meta-analysis. Can J Neurol Sci J Can Sci Neurol 43:S3-S50. https://doi.org/10.1017/cjn.2016.18

4. Nordon IM, Hinchliffe RJ, Loftus IM, Thompson MM (2011) Pathophysiology and epidemiology of abdominal aortic aneurysms. Nat Rev Cardiol 8:92-102. https://doi.org/10.1038/nrcardio.2010.180

5. Wang J-C, Chien W-C, Tzeng N-S, et al (2019) Surgical repair of aortic aneurysms and reduced incidence of dementia. Int J Cardiol 278:46-50. https://doi.org/10.1016/j.ijcard.2018.11.137

6. Tisher A, Salardini A (2019) A Comprehensive Update on Treatment of Dementia. Semin Neurol 39:167-178. https://doi.org/10.1055/s-00391683408

7. Tublin JM, Adelstein JM, del Monte F, et al (2019) Getting to the Heart of Alzheimer Disease. Circ Res 124:142-149. https://doi.org/10.1161/CIRCRESAHA.118.313563

8. Lee Y, Han K, Ko S-H, et al (2016) Data Analytic Process of a Nationwide Population-Based Study Using National Health Information Database Established by National Health Insurance Service. Diabetes Metab J 40:79. https://doi.org/10.4093/dmj.2016.40.1.79

9. Wimo A, Jönsson L, Bond J, et al (2013) The worldwide economic impact of dementia 2010. Alzheimers Dement 9:1-11.e3. https://doi.org/10.1016/j.jalz.2012.11.006

10. Perng $\mathrm{C}-\mathrm{H}$, Chang $\mathrm{Y}-\mathrm{C}$, Tzang R-F (2018) The treatment of cognitive dysfunction in dementia: a multiple treatments meta-analysis. Psychopharmacology (Berl) 235:1571-1580. https://doi.org/10.1007/s00213-018-4867-y

11. Biessels GJ, Despa F (2018) Cognitive decline and dementia in diabetes mellitus: mechanisms and clinical implications. Nat Rev Endocrinol 14:591-604. https://doi.org/10.1038/s41574-018-0048-7

12. Bello-Chavolla OY, Antonio-Villa NE, Vargas-Vázquez A, et al (2019) Pathophysiological Mechanisms Linking Type 2 Diabetes and Dementia: Review of Evidence from Clinical, Translational and Epidemiological Research. Curr Diabetes Rev 15:456-470. https://doi.org/10.2174/1573399815666190129155654

13. Walker KA, Power MC, Gottesman RF (2017) Defining the Relationship Between Hypertension, Cognitive Decline, and Dementia: a Review. Curr Hypertens Rep 19:24. https://doi.org/10.1007/s11906-017-0724-3

14. Roher A, Debbins, Malek-Ahmadi M, et al (2012) Cerebral blood flow in Alzheimer\&rsquo;s disease. Vasc Health Risk Manag 599. https://doi.org/10.2147/VHRM.S34874

15. Farruggia MC, Small DM (2019) Effects of adiposity and metabolic dysfunction on cognition: A review. Physiol Behav 208:112578. https://doi.org/10.1016/j.physbeh.2019.112578 
16. Durazzo TC, Mattsson N, Weiner MW, Alzheimer's Disease Neuroimaging Initiative (2014) Smoking and increased Alzheimer's disease risk: A review of potential mechanisms. Alzheimers Dement 10:S122-S145. https://doi.org/10.1016/j.jalz.2014.04.009

17. Raffort J, Lareyre F, Clément M, et al (2018) Diabetes and aortic aneurysm: current state of the art. Cardiovasc Res 114:1702-1713. https://doi.org/10.1093/cvr/cvy174

18. Kobeissi E, Hibino M, Pan H, Aune D (2019) Blood pressure, hypertension and the risk of abdominal aortic aneurysms: a systematic review and meta-analysis of cohort studies. Eur J Epidemiol 34:547-555. https://doi.org/10.1007/s10654-019-00510-9

19. Liu C-L, Ren J, Wang Y, et al (2020) Adipocytes promote interleukin-18 binding to its receptors during abdominal aortic aneurysm formation in mice. Eur Heart J 41:2456-2468. https://doi.org/10.1093/eurheartj/ehz856

20. Jin J, Arif B, Garcia-Fernandez F, et al (2012) Novel Mechanism of Aortic Aneurysm Development in Mice Associated With Smoking and Leukocytes. Arterioscler Thromb Vasc Biol 32:2901-2909. https://doi.org/10.1161/ATVBAHA.112.300208

21. Golledge $\mathrm{J}$ (2019) Abdominal aortic aneurysm: update on pathogenesis and medical treatments. Nat Rev Cardiol 16:225-242. https://doi.org/10.1038/s41569-018-0114-9

22. Golledge J, Norman PE (2010) Atherosclerosis and Abdominal Aortic Aneurysm: Cause, Response, or Common Risk Factors? Arterioscler Thromb Vasc Biol 30:1075-1077. https://doi.org/10.1161/ATVBAHA.110.206573

23. Pasterkamp G, Strauss BH, de Kleijn D (2007) Arterial Remodeling. In: Duckers HJ, Nabel EG, Serruys PW (eds) Essentials of Restenosis. Humana Press, Totowa, NJ, pp 213-225

24. Yao L, Folsom AR, Alonso A, et al (2018) Association of carotid atherosclerosis and stiffness with abdominal aortic aneurysm: The atherosclerosis risk in communities (ARIC) study. Atherosclerosis 270:110-116. https://doi.org/10.1016/j.atherosclerosis.2018.01.044

25. Muhire G, lulita MF, Vallerand D, et al (2019) Arterial Stiffness Due to Carotid Calcification Disrupts Cerebral Blood Flow Regulation and Leads to Cognitive Deficits. J Am Heart Assoc 8:. https://doi.org/10.1161/JAHA.118.011630

26. Aparicio HJ, Petrea RE, Massaro JM, et al (2017) Association of descending thoracic aortic plaque with brain atrophy and white matter hyperintensities: The Framingham Heart Study. Atherosclerosis 265:305-311. https://doi.org/10.1016/j.atherosclerosis.2017.06.919

27. van der Veen PH, Muller M, Vincken KL, et al (2014) Longitudinal changes in brain volumes and cerebrovascular lesions on MRI in patients with manifest arterial disease: The SMART-MR study. J Neurol Sci 337:112-118. https://doi.org/10.1016/j.jns.2013.11.029

28. Sakalihasan N, Limet R, Defawe OD (2005) Abdominal aortic aneurysm. 365:13

29. Tamarina NA, McMillan WD, Shively VP, Pearce WH (1997) Expression of matrix metalloproteinases and their inhibitors in aneurysms and normal aorta. Surgery 122:264-272. https://doi.org/10.1016/S0039-6060(97)90017-9

30. Allaire E, Muscatelli-Groux B, Mandet C, et al (2002) Paracrine effect of vascular smooth muscle cells in the prevention of aortic aneurysm formation. J Vasc Surg 36:1018-1026. https://doi.org/10.1067/mva.2002.127347

31. Wang H, Huang L, Wu L, et al (2020) The MMP-2/TIMP-2 System in Alzheimer Disease. CNS Neurol Disord - Drug Targets 19:402-416. https://doi.org/10.2174/1871527319666200812223007

32. Tuna G, Yener GG, Oktay G, et al (2018) Evaluation of Matrix Metalloproteinase-2 (MMP-2) and -9 (MMP-9) and Their Tissue Inhibitors (TIMP-1 and TIMP-2) in Plasma from Patients with Neurodegenerative Dementia. J Alzheimers Dis 66:1265-1273. https://doi.org/10.3233/JAD180752

33. Cheignon C, Tomas M, Bonnefont-Rousselot D, et al (2018) Oxidative stress and the amyloid beta peptide in Alzheimer's disease. Redox Biol 14:450-464. https://doi.org/10.1016/j.redox.2017.10.014

\section{Figures}




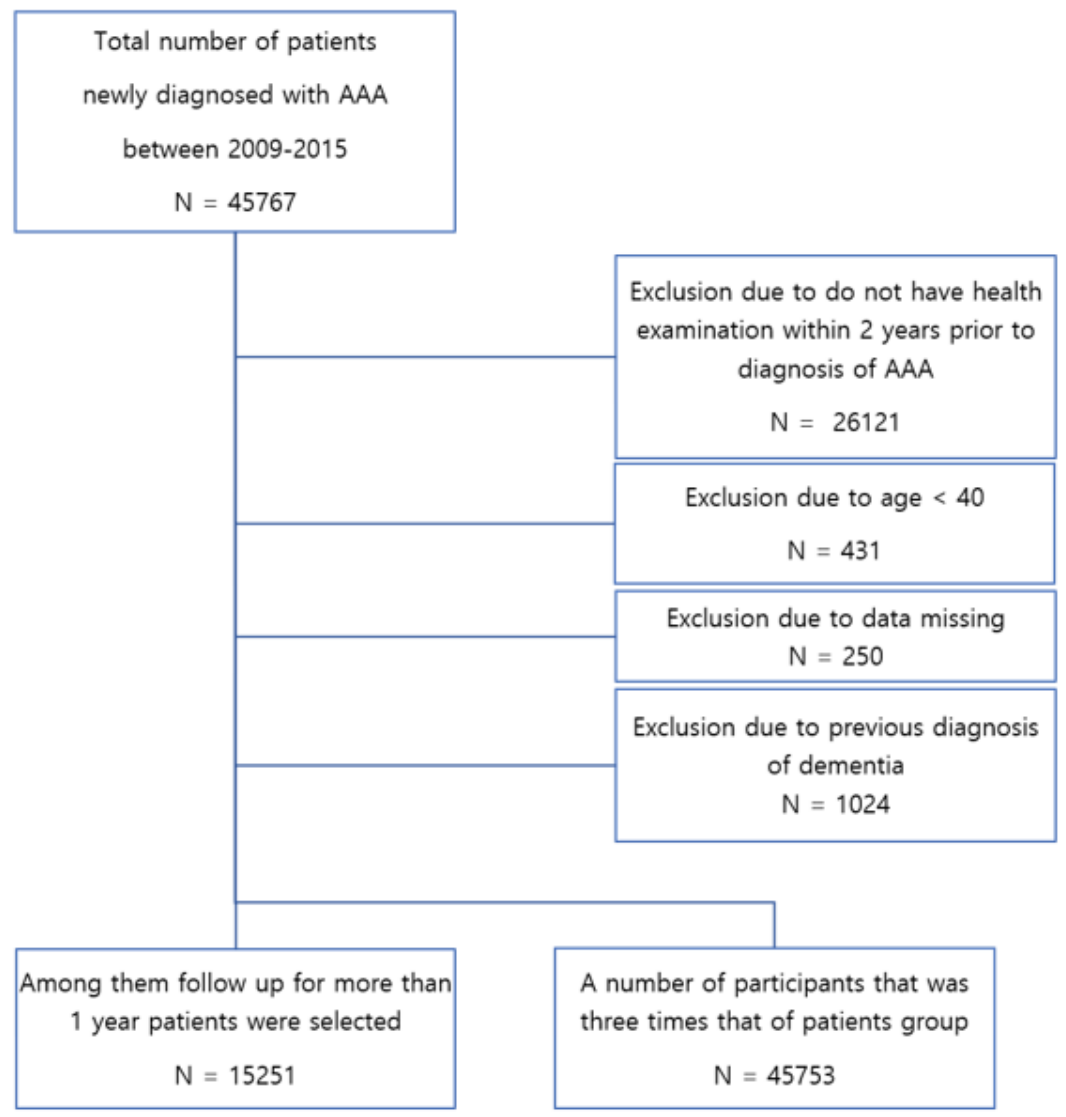

\section{Figure 1}

Enrollment flow chart. 

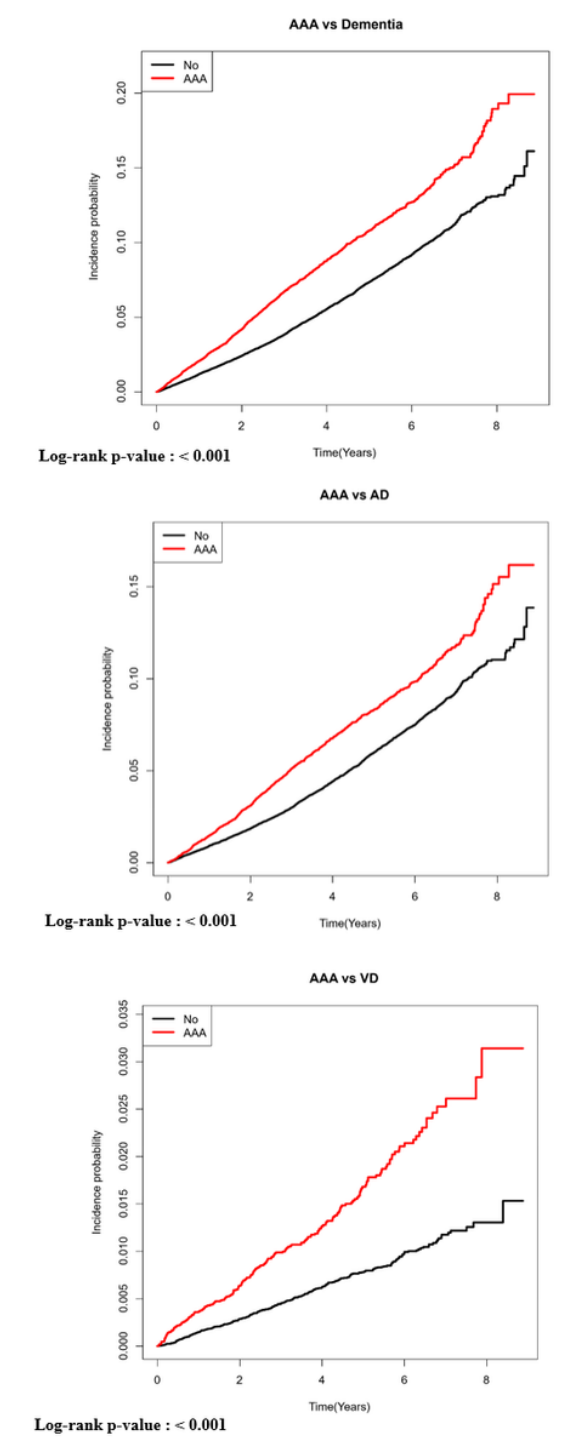

\section{Figure 2}

Kaplan-Meier plot for incidence of dementia in patients with AAA

\section{Supplementary Files}

This is a list of supplementary files associated with this preprint. Click to download.

- SupplementalTable.docx 